\title{
High prevalence of non-alcoholic fatty liver disease in patients with inflammatory bowel disease receiving anti-tumor necrosis factor therapy
}

\author{
Alisa Likhitsup ${ }^{a, b}$, Jason Dundulis ${ }^{a, b}$, Shaya Ansaria, ${ }^{a, b}$, Sruthi Patibandla ${ }^{a}$, Colleen Hutton ${ }^{c}$, \\ Kevin Kennedyc, John H. Helzberga,b, Rajiv Chhabra ${ }^{a, b}$
}

University of Missouri Kansas City; Saint Luke's Hospital of Kansas City; Mid-America Heart Institute St. Luke’s Health System, Kansas City, MO, USA

\section{Abstract}

${ }^{a}$ University of Missouri Kansas City (Alisa Likhitsup, Jason Dundulis, Shaya Ansari, Sruthi Patibandla, John H. Helzberg, Rajiv Chhabra); 'Saint Luke's Hospital of Kansas City (Alisa Likhitsup, Jason Dundulis, Shaya Ansari, John H. Helzberg, Rajiv Chhabra); 'Mid-America Heart Institute St. Luke's Health System (Colleen Hutton, Kevin Kennedy), Kansas City, MO, USA

Conflict of Interest: Dr. John H. Helzberg received research funding from Henry and Marion Bloch Liver Disease Management Fund. Other authors have nothing to disclose

Correspondence to: Alisa Likhitsup, MD, 4320 Wornall Rd, Suit 240, Kansas City, MO 64111, USA, e-mail: alikhitsup@gmail.com

Received 28 April 2019; accepted 25 June 2019; published online 22 July 2019

DOI: https://doi.org/10.20524/aog.2019.0405

\section{Introduction}

Non-alcoholic fatty liver disease (NAFLD) is more common in the western world [1]. In the general US population, the prevalence of NAFLD has been reported to be $19-46 \%$ [2-13]. Risk factors for NAFLD include obesity, insulin resistance, diabetes mellitus, hypertension, and hypertriglyceridemia [14]. The spectrum of NAFLD is broad and ranges from benign macrovesicular steatosis to advanced steatohepatitis, cirrhosis, and liver cancer. NAFLD has become more commonly recognized among patients with inflammatory bowel disease (IBD). The prevalence of NAFLD in IBD patients has been reported to vary from $8-59 \%$, depending on the diagnostic criteria used [15-20]. IBD, which includes Crohn's disease (CD) and ulcerative colitis (UC), is a chronic inflammatory disease of 
the gastrointestinal tract with an estimated prevalence of 1-1.3 million in the US [21]. The pathogenesis of NAFLD among the IBD population is not well understood. Apart from metabolic syndrome, IBD-specific risk factors include malnutrition, intestinal inflammation, gut microbiota alteration, steroid exposure, and drug-induced hepatotoxicity, all of which may contribute to the pathogenesis of NAFLD [22].

The effect of anti-tumor necrosis factor (TNF) therapy on NAFLD development in IBD patients remains unclear $[15,16]$. The aim of this study was to evaluate the prevalence of NAFLD among IBD patients receiving anti-TNF therapy. Predictive factors associated with NAFLD among IBD patients were evaluated. The prevalence of steatohepatitis and hepatic fibrosis among IBD patients with NAFLD was assessed.

\section{Patients and methods}

The Institutional Review Board of Saint Luke's Hospital of Kansas City approved the data collection and methodology of this study. The study was performed in accordance with relevant guidelines. Informed consent was obtained from all participants. We conducted a cross-sectional study that included IBD patients, aged 18 years or older, who had been receiving anti-TNF therapy (infliximab, adalimumab, certolizumab, golimumab) through the Saint Luke's gastrointestinal specialist and Mid-America Gastrointestinal consultants ambulatory clinic for at least 6 months prior to study enrollment. Study questionnaires were completed and the following data were collected: age, body mass index (BMI), waist circumference, IBD history including disease activity, and risk factors for NAFLD (Supplementary Table 1). Blood samples were obtained for a comprehensive metabolic panel, viral hepatitis and iron studies (to exclude chronic viral hepatitis and hemochromatosis). Patients with a history of concurrent hepatitis B or C infection, elevated iron saturation $>45 \%$, excessive alcohol use (defined as $>7$ alcoholic drinks/ week in women or $>14$ alcoholic drinks/week in men) or recent steroid use within the past 6 months were excluded. Abdominal ultrasound examinations were completed and reviewed by a specialized abdominal radiologist (SA) for the presence or absence of hepatic steatosis. A non-alcoholic steatohepatitis (NASH) FibroSure (LabCorp, Burlington, NC) test was completed in those patients with evidence of hepatic steatosis on abdominal ultrasound to estimate the degree of inflammation and fibrosis.

\section{Statistical analysis}

The data were presented as mean \pm standard deviation for continuous variables and as a total percentage for categorical variables. Student's $t$-test and chi-square tests were used in the unadjusted analysis. The prevalence of NAFLD was compared to that of the general US population, represented by a national database [1]. Assuming a prevalence of $30 \%$, we required
80 patients to ensure that a $95 \%$ confidence interval $(95 \% \mathrm{CI})$ estimate of the proportion of IBD patients with NAFLD is within $10 \%$ of the true population prevalence. The prevalence of hepatic steatosis, steatohepatitis and fibrosis was assessed by NASH FibroSure (LabCorp, Burlington, NC). To assess the predictive factors associated with the presence of NAFLD in IBD patients, a multivariate logistic regression model was used, adjusted for age, sex, weight, BMI, waist circumference, presence of diabetes mellitus, and transaminase levels. SPSS version 21.0 (IBM Corp, Armonk, NY) was used for all analyses.

\section{Results}

\section{NAFLD prevalence in IBD patients receiving anti-TNF therapy}

Eighty patients were enrolled, with a mean age of $42 \pm 15$ years, predominantly male $(55 \%)$ and with average BMI $26 \pm 5.7 \mathrm{~kg} / \mathrm{m}^{2}$. Sixty-five patients (81\%) had CD, with mean CD activity index (CDAI) $28 \pm 25$, while 15 patients (19\%) had UC, with mean simple clinical colitis activity index (SCCAI) $2 \pm 2.15$ (Table 1). The mean duration of IBD since diagnosis was $14 \pm 10$ years and the mean duration of antiTNF therapy was $5 \pm 4$ years. The numbers of patients receiving adalimumab, infliximab, golimumab, and certolizumab therapy were $67(84 \%), 10(13 \%), 2(2.5 \%)$, and $1(1.3 \%)$, respectively. Abdominal ultrasound detected hepatic steatosis in 43 patients, giving a prevalence of NAFLD in these patients of 54\%' significantly higher than that of the general US population, assuming a prevalence of $30 \%(\mathrm{P}<0.001)$.

\section{Univariate comparison of IBD patients with and without NAFLD}

IBD patients with hepatic steatosis had a higher percentage of male sex ( $67 \%$ vs. $41 \%$; $\mathrm{P}=0.02)$, with greater body weight ( $84 \pm 20$ vs. $69 \pm 18 \mathrm{~kg} ; \mathrm{P}=0.001)$ and BMI $\left(30 \pm 6\right.$ vs. $24 \pm 4 \mathrm{~kg} / \mathrm{m}^{2}$; $\mathrm{P}=0.001$ ). Among patients with $\mathrm{CD}$, those with NAFLD had a higher mean CDAI score ( $35 \pm 27$ vs. $22 \pm 24 ; \mathrm{P}=0.01)$. There was no difference between the NAFLD and non-NAFLD groups in age, type of IBD (CD vs. UC), duration of IBD, duration and type of anti-TNF therapy, type of concurrent IBD medications, waist circumference, presence of diabetes, transaminase levels, or SSCCI scores (in UC patients) (Table 1).

\section{Multivariate analysis of predictive factors for NAFLD}

In the multivariate logistic regression model, age, sex, weight, BMI, waist circumference, presence of diabetes mellitus, and transaminase levels were included in the final model. An increase in CDAI score was associated with a 1.6 times higher likelihood of NAFLD development (Table 2). 
Table 1 Characteristic of the study populations

\begin{tabular}{|c|c|c|c|c|}
\hline \multirow[t]{2}{*}{ Characteristics } & \multirow{2}{*}{$\begin{array}{c}\text { Total } \\
(\mathrm{n}=80)\end{array}$} & \multicolumn{2}{|c|}{ Hepatic steatosis } & \multirow[t]{2}{*}{ P-value } \\
\hline & & Yes $(n=43)$ & No $(n=37)$ & \\
\hline Age (years) & $42 \pm 15$ & $45 \pm 14$ & $40 \pm 16$ & 0.13 \\
\hline Male & $44(55 \%)$ & $29(67 \%)$ & $15(41 \%)$ & $0.02^{*}$ \\
\hline Weight (kg) & $77 \pm 20$ & $84 \pm 20$ & $69 \pm 18$ & $0.001^{\star}$ \\
\hline BMI $\left(\mathrm{kg} / \mathrm{cm}^{2}\right)$ & $26 \pm 5.7$ & $30 \pm 6$ & $24 \pm 4$ & $0.001^{\star}$ \\
\hline Waist circumference (inches) & $33.4 \pm 5.4$ & $35 \pm 5$ & $32 \pm 5$ & 0.17 \\
\hline Crohn's disease (n, \%) & $65(81 \%)$ & $37(86 \%)$ & $28(76 \%)$ & 0.24 \\
\hline History of diabetes mellitus & $3(4 \%)$ & $3(7 \%)$ & 0 & 0.10 \\
\hline AST (IU/L) & $35 \pm 16$ & $36 \pm 17$ & $33 \pm 16$ & 0.39 \\
\hline ALT (IU/L) & $38 \pm 22$ & $42 \pm 25$ & $34 \pm 18$ & 0.12 \\
\hline Duration of IBD diagnosis (years) & $14 \pm 10$ & $15 \pm 10$ & $13 \pm 11$ & 0.69 \\
\hline Duration of biologic therapy (years) & $5 \pm 4$ & $5 \pm 5$ & $4 \pm 4$ & 0.71 \\
\hline Biologic therapy & & & & 0.82 \\
\hline Infliximab & $67(84 \%)$ & $36(84 \%)$ & $31(84 \%)$ & \\
\hline Adalimumab & $10(13 \%)$ & $5(12 \%)$ & $5(14 \%)$ & \\
\hline Golimumab & $2(2.5 \%)$ & $1(2.3 \%)$ & $1(2.7 \%)$ & \\
\hline Certolizumab & $1(1.3 \%)$ & $1(2.3 \%)$ & 0 & \\
\hline Concurrent IBD medications & & & & 0.46 \\
\hline Sulfasalazine/Mesalamine & $12(15 \%)$ & $7(16 \%)$ & $5(14 \%)$ & \\
\hline AZA/6-MP & $17(21 \%)$ & $10(23 \%)$ & $7(19 \%)$ & \\
\hline Methotrexate & $2(2.5 \%)$ & 0 & $2(5.4 \%)$ & \\
\hline CDAI in CD $(n=65)$ & $28 \pm 25$ & $35 \pm 27$ & $22 \pm 24$ & $0.01^{*}$ \\
\hline SCCAI in UC $(n=15)$ & $2 \pm 2.15$ & $1.5 \pm 0.7$ & $2.5 \pm 2.8$ & 0.25 \\
\hline
\end{tabular}

${ }^{*}$ Alpha less than 0.05 considered statistically significant

$A L T$, alanine aminotransferase; AST, aspartate aminotransferase; $A Z A$, azathioprine; BMI, body mass index; $C D$, Crohn's disease; CDAI, Crohn's disease activity index; lbs, pounds; 6-MP, 6-mercaptopurine; SCCAI, simple clinical colitis activity index; UC, ulcerative colitis

\section{Prevalence of NASH and hepatic fibrosis in IBD patients with NAFLD}

In patients with hepatic steatosis detected on abdominal ultrasound, 31 of 43 patients (72\%) completed a NASH Fibrosure test. Of these 31 patients, 21 (67\%) had steatohepatitis, 19 (61\%) had steatohepatitis, and 7 (22\%) had hepatic fibrosis (16\% with portal fibrosis, $3 \%$ with bridging fibrosis with few septa, and 3\% with bridging fibrosis with many septa) (Fig. 1). No cirrhosis was detected based on NASH Fibrosure or abdominal ultrasound.

\section{Discussion}

Because of the obesity epidemic and the increasing prevalence of metabolic syndrome, the global prevalence of NAFLD has been rising to an estimated level of $24 \%$ [1]. In the general US population, the reported sonographic and biopsy-proven prevalences of NAFLD were $19-34 \%$ and 34-46\%, respectively [2-13].

The prevalence of NAFLD in IBD patients has been reported to vary from $8-59 \%$, depending on the diagnostic criteria used [15-20]. In one study, 108 of 321 IBD patients (34\%) were diagnosed with NAFLD based on the hepatic steatosis index, with a reported annual incidence of 9.1\% [15]. However, a serology-based diagnosis of NAFLD can lead to underreporting of its true prevalence. Ultrasonography is a widely accessible imaging technique for the detection of hepatic steatosis, with high sensitivity $(85 \%, 95 \%$ CI $79.5-88.9 \%)$, specificity (94\%, 95\%CI 87.2-97\%) and positive likelihood ratio (13.3, 95\% CI 6.427.6) compared to histology [23]. The sonographic prevalence of hepatic steatosis was $26-40 \%$ in CD and $26-36 \%$ in UC patients without obesity, metabolic disorders, or underlying chronic hepatitis [18]. Another retrospective study reported only 76 of 928 (8\%) IBD patients had evidence of NAFLD based on various 


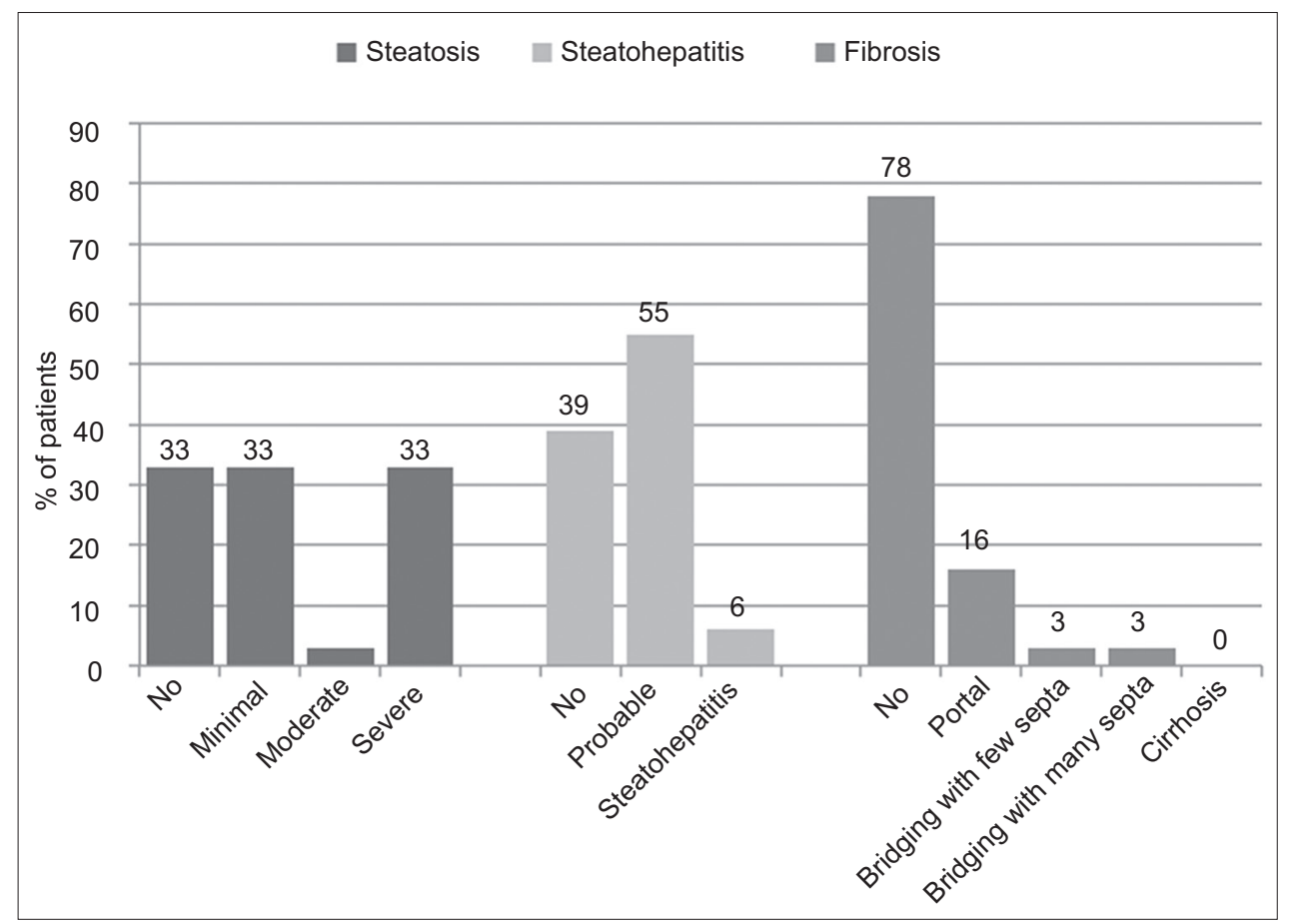

Figure 1 NASH Fibrosure in patients with the hepatic steatosis $(\mathrm{n}=31)$

Steatosis score; $\mathrm{S} 0=<0.3$ (no steatosis), $\mathrm{S} 0-1=0.3-0.4$ (minimal steatosis), $\mathrm{S} 1-2=0.48-0.67$ (moderate steatosis), $\mathrm{S} 2-3 \geq 0.67$ (severe steatosis). Steatohepatitis score; $\mathrm{N} 0=0.25$ (no steatohepatitis), $\mathrm{N} 1=0.50$ (probable steatohepatitis), $\mathrm{N} 2=0.75$ (steatohepatitis). Fibrosis score; F0 0.21 (no fibrosis), F0-1=0.21-0.30 (portal fibrosis), F1-2=0.31-0.58 (bridging fibrosis with few septa), F3=0.58-0.72 (bridging fibrosis with many septa), F4 $\geq 0.72$ (cirrhosis)

types of imaging studies [16]. Histological diagnosis is the gold standard, but it is invasive, as a tissue specimen is needed for the diagnosis. Fifty-nine percent of IBD patients were found to have at least grade-1 hepatic steatosis on liver histology (from liver biopsy, resection, or autopsy) [17]. In our study, the prevalence of NAFLD in IBD patients who had received anti-TNF therapy for a minimum of 6 months without steroid exposure was 54\%, based on abdominal ultrasound.

Among IBD patients, $20-30 \%$ of children and $18 \%$ of adults are obese and the prevalence is rising [24]; 38\% of adults with IBD were overweight [25]. Hypertension, obesity and metabolic syndrome were reportedly associated with the presence of NAFLD in IBD patients $[16,19]$. In our crosssectional cohort, patients with NAFLD had higher body weight and BMI. There was no difference in waist circumference or the presence of diabetes.

Several studies have suggested that the duration of IBD is associated with the development of NAFLD [15,26]. Patients with a longer disease duration are potentially exposed to multiple risk factors associated with NAFLD, including chronic inflammation, intestinal permeability, alteration of gut microbiota, metabolic comorbidities, and hepatotoxic drugs [15]. In our study, in which the patients' mean IBD duration was $14 \pm 10$ years, we did not detect any association between IBD duration and NAFLD.

A retrospective study of $321 \mathrm{IBD}$ patients reported disease activity associated with NAFLD detected by the hepatic steatosis index as having a hazard ratio of 1.58 (95\%CI 1-08-
2.33; $\mathrm{P}=0.02)$ [15]. The IBD disease severity was found to be associated with elevated hepatic fat content detected on ultrasonography [27]. Another study in patients with UC reported that liver fat content might be associated with UC severity score [28]. Prior small bowel surgery, which implicitly reflects the IBD disease severity, has also been associated with NAFLD [15]. In contrast, some studies reported no such association between NAFLD and IBD status, disease activity or drugs $[19,29]$. In our study, the CDAI, but not the SSCCI, was found to be associated with the development of NAFLD. The multivariate logistic regression analysis showed that a high CDAI score was independently associated with a 1.6 times higher likelihood of NAFLD development. We did not detect any association between treatment duration, type of biologic therapy or concurrent IBD medication with NAFLD (Table 1).

Proinflammatory cytokines such as interleukins, TNF- $\alpha$ and adhesion factors, contribute to chronic inflammation and IBD exacerbation, which also have hepatic effects. TNF- $\alpha$ is able to trigger stellate cell activation, matrix gene expression and remodeling, important events during the onset of steatohepatitis [30]. In an experimental rat model, TNF inhibition reduced hepatic fat content, inflammation, necrosis and fibrosis, and improved insulin signal transduction in treated animals compared with placebo [31,32]. Pentoxifylline (PTX) inhibits a number of proinflammatory cytokines, including TNF- $\alpha$. PTX has been studied in a NASH population and achieved an improvement in NASH histological features compared to placebo [33]. A meta-analysis showed that PTX reduced 
Table 2 Predictive factors associated with the presence of nonalcoholic fatty liver disease in inflammatory bowel disease patients who received biologic treatment $(\mathrm{n}=80)$

\begin{tabular}{|c|c|c|}
\hline Variables & $\mathrm{OR}^{\star}(95 \% \mathrm{CI})$ & P-value \\
\hline Male sex & $3.1(0.7-12.5)$ & 0.11 \\
\hline Increased weight & $0.9(.97-1.03)$ & 0.85 \\
\hline Increased BMI & $1.2(0.9-1.5)$ & 0.17 \\
\hline Increased CDAI score & $1.6(1.05-2.44)$ & $0.03^{* *}$ \\
\hline \multicolumn{3}{|c|}{$\begin{array}{l}\text { *Adjusted for age, sex, weight, BMI, waist circumference, presence of } \\
\text { diabetes mellitus, and transaminase levels }\end{array}$} \\
\hline \multicolumn{3}{|c|}{${ }^{* *}$ Alpha less than 0.05 considered statistically significant } \\
\hline \multicolumn{3}{|c|}{$\begin{array}{l}\text { BMI, body mass index; CDAI, Crohn's disease activity index; OR, odd ratio; } \\
\text { CI, confidence interval }\end{array}$} \\
\hline
\end{tabular}

aspartate aminotransferase and alanine aminotransferase levels and improves histological scores in patients with NAFLD and NASH, but did not affect cytokines [34]. Anti-TNF therapy has become more commonly used in IBD patients. Bessisow et al demonstrated that anti-TNF therapy was not associated with NAFLD in IBD patients [15]. In contrast, Sourianarayanane et al suggested that anti-TNF was protective against NAFLD [16]. However, these results should be interpreted cautiously, as the study included patients concurrently using steroids. Another meta-analysis showed that the pooled odds ratio [OR] for NAFLD in IBD patients using a biologic agent was 0.85 (95\%CI 0.49-1.46) [35]. Another study reported that ongoing antiTNF therapy was an independent protective factor against transaminase abnormalities (OR 0.15, 95\%CI, 0-0.8; $\mathrm{P}=0.02$ ) [27]. In our study, $54 \%$ of IBD patients who had received anti-TNF therapy for a mean duration of $5 \pm 4$ years developed hepatic steatosis based on abdominal ultrasound. Hence, there may be factors other than the cytokine-mediated pathway that contribute to the pathogenesis of NAFLD in the IBD population.

The annual incidence of advanced liver fibrosis among IBD patients was $0.5 \%$ (0.2-1.1) detected by FIB- 4 score [15]; $6.4 \%$ of IBD patients had elevated liver stiffness measurements $(>8 \mathrm{kPa})$ detected by transient elastography and 3 patients $(2.7 \%)$ had persistently elevated liver stiffness ( $>8 \mathrm{kPa}) 6$ months later [36]. The NASH FibroSure test (LabCorp, Burlington, NC) is a noninvasive assessment of hepatic steatosis, with a sensitivity and specificity of $46-90 \%$ and $54-88 \%$, respectively, in the detection of hepatic steatosis and a positive predictive value of $46-63 \%$ [37]. The test performed better in the detection of steatohepatitis (sensitivity $30-88 \%$, specificity $50-94 \%$, positive predictive value $66-74 \%$ ). The sensitivity and specificity in detecting advanced hepatic fibrosis were $25-92 \%$ and $71-97 \%$, respectively, with a positive predictive value of 33-60\% [38,39].

In our study, NASH FibroSure was applied only in patients who had hepatic steatosis based on abdominal ultrasound: $61 \%$ of the patients had hepatic steatosis (55\% probable and $6 \%$ definite), while $22 \%$ of patients had hepatic fibrosis (6\% with bridging fibrosis) (Fig. 1). None of our patients was diagnosed with cirrhosis.

The limitations of our study are that it was a cross-sectional cohort study, without a control population to evaluate the predictive factors associated with the development or progression of NAFLD. Given our limited resources, we did not collect C-reactive protein, fecal calprotectin or serum TNF drug levels. Furthermore, our patients were receiving anti-TNF therapy and their IBD activity was well controlled. Thus, the NAFLD prevalence yielded by our study will not apply to IBD patients with uncontrolled IBD, or to those who are not receiving antiTNF therapy or are actively on steroids. Furthermore, the sample size was too small to perform subgroup analysis for CD and UC separately. Although the prevalence of fatty liver is known to be elevated among IBD populations, additional studies are still needed to establish the benefit of NAFLD screening, risk factors associated with NAFLD development and predictive factors associated with NAFLD progression among IBD populations.

In conclusion, the presence of IBD strongly associated with NAFLD. Our cross-sectional study identified a high prevalence of NAFLD among IBD patients receiving anti-TNF therapy. As to whether IBD disease activity is associated with NAFLD, the data are conflicting. Our study results showed that CD severity, but not UC disease severity, was independently associated with hepatic steatosis. These data suggest that, apart from changes in the prevalence of metabolic disease, there are IBD-associated factors that contribute to the pathogenesis of NAFLD among IBD population. However, further studies are still needed.

\section{Summary Box}

\section{What is already known:}

- Risk factors for non-alcoholic fatty liver disease (NAFLD) include obesity, insulin resistance, diabetes mellitus, hypertension, and hypertriglyceridemia

- The prevalence of NAFLD in patients with inflammatory bowel disease (IBD) reportedly varies from $8-59 \%$, depending on the diagnostic criteria

- There are conflicting data regarding the effect of anti-tumor necrosis factor (TNF) therapy on NAFLD prevalence among IBD populations

\section{What the new findings are:}

- The presence of IBD is strongly associated with NAFLD

- We identified a high prevalence of NAFLD among IBD patients receiving anti-TNF therapy

- There are probably IBD-associated factors that contribute to the pathogenesis of NAFLD among IBD population, but further studies are still needed

\section{References}

1. Younossi ZM, Koenig AB, Abdelatif D, Fazel Y, Henry L, Wymer M. Global epidemiology of nonalcoholic fatty liver disease-metaanalytic assessment of prevalence, incidence, and outcomes. Hepatology 2016;64:73-84.

2. Lazo M, Hernaez R, Bonekamp S, et al. Non-alcoholic fatty liver 
disease and mortality among US adults: prospective cohort study. BMJ 2011;343:d6891.

3. Younossi ZM, Stepanova M, Negro F, et al. Nonalcoholic fatty liver disease in lean individuals in the United States. Medicine (Baltimore) 2012;91:319-327.

4. Kim D, Kim WR, Kim HJ, Therneau TM. Association between noninvasive fibrosis markers and mortality among adults with nonalcoholic fatty liver disease in the United States. Hepatology 2013;57:1357-1365.

5. Lazo M, Hernaez R, Eberhardt MS, et al. Prevalence of nonalcoholic fatty liver disease in the United States: the Third National Health and Nutrition Examination Survey, 1988-1994. Am J Epidemiol 2013; 178:38-45.

6. Ruhl CE, Everhart JE. Relationship of non-alcoholic fatty liver disease with cholecystectomy in the US population. Am J Gastroenterol 2013;108:952-958.

7. Otgonsuren M, Stepanova M, Gerber L, Younossi ZM. Anthropometric and clinical factors associated with mortality in subjects with nonalcoholic fatty liver disease. Dig Dis Sci 2013;58:1132-1140.

8. Younossi ZM, Otgonsuren M, Venkatesan C, Mishra A. In patients with non-alcoholic fatty liver disease, metabolically abnormal individuals are at a higher risk for mortality while metabolically normal individuals are not. Metabolism 2013;62:352-360.

9. Smits MM, Ioannou GN, Boyko EJ, Utzschneider KM. Nonalcoholic fatty liver disease as an independent manifestation of the metabolic syndrome: results of a US national survey in three ethnic groups. J Gastroenterol Hepatol 2013;28:664-670.

10. Schneider AL, Lazo M, Selvin E, Clark JM. Racial differences in nonalcoholic fatty liver disease in the U.S. population. Obesity (Silver Spring) 2014;22:292-299.

11. Mohanty SR, Troy TN, Huo D, O’Brien BL, Jensen DM, Hart J. Influence of ethnicity on histological differences in non-alcoholic fatty liver disease. J Hepatol 2009;50:797-804.

12. Setji TL, Holland ND, Sanders LL, Pereira KC, Diehl AM, Brown AJ. Nonalcoholic steatohepatitis and nonalcoholic fatty liver disease in young women with polycystic ovary syndrome. J Clin Endocrinol Metab 2006;91:1741-1747.

13. Williams CD, Stengel J, Asike MI, et al. Prevalence of nonalcoholic fatty liver disease and nonalcoholic steatohepatitis among a largely middle-aged population utilizing ultrasound and liver biopsy: a prospective study. Gastroenterology 2011;140:124-131.

14. Chalasani N, Younossi Z, Lavine JE, et al. The diagnosis and management of nonalcoholic fatty liver disease: Practice guidance from the American Association for the Study of Liver Diseases. Hepatology 2018;67:328-357.

15. Bessissow T, Le NH, Rollet K, Afif W, Bitton A, Sebastiani G. Incidence and predictors of nonalcoholic fatty liver disease by serum biomarkers in patients with inflammatory bowel disease. Inflamm Bowel Dis 2016;22:1937-1944.

16. Sourianarayanane A, Garg G, Smith TH, Butt MI, McCullough AJ, Shen B. Risk factors of non-alcoholic fatty liver disease in patients with inflammatory bowel disease. JCrohns Colitis 2013;7:e279-e285.

17. Bosch DE, Yeh MM. Primary sclerosing cholangitis is protective against nonalcoholic fatty liver disease in inflammatory bowel disease. Hum Pathol 2017;69:55-62.

18. Bargiggia S, Maconi G, Elli M, et al. Sonographic prevalence of liver steatosis and biliary tract stones in patients with inflammatory bowel disease: study of 511 subjects at a single center. J Clin Gastroenterol 2003;36:417-420.

19. Carr RM, Patel A, Bownik H, et al. Intestinal inflammation does not predict nonalcoholic fatty liver disease severity in inflammatory bowel disease patients. Dig Dis Sci 2017;62:1354-1361.

20. Likhitsup A, Dundulis J, Ansari S, et al. Prevalence of non-alcoholic fatty liver disease on computed tomography in patients with inflammatory bowel disease visiting an emergency department.
Ann Gastroenterol 2019;32:283-286.

21. Kappelman MD, Rifas-Shiman SL, Kleinman K, et al. The prevalence and geographic distribution of Crohn's disease and ulcerative colitis in the United States. Clin Gastroenterol Hepatol 2007;5:1424-1429.

22. Miele L, Valenza V, La Torre G, et al. Increased intestinal permeability and tight junction alterations in nonalcoholic fatty liver disease. Hepatology 2009;49:1877-1887.

23. Hernaez R, Lazo M, Bonekamp S, et al. Diagnostic accuracy and reliability of ultrasonography for the detection of fatty liver: a meta-analysis. Hepatology 2011;54:1082-1090.

24. Long MD, Crandall WV, Leibowitz IH, et al; ImproveCareNow Collaborative for Pediatric IBD. Prevalence and epidemiology of overweight and obesity in children with inflammatory bowel disease. Inflamm Bowel Dis 2011;17:2162-2168.

25. Steed H, Walsh S, Reynolds N. A brief report of the epidemiology of obesity in the inflammatory bowel disease population of Tayside, Scotland. Obes Facts 2009;2:370-372.

26. Glassner K, Malaty HM, Abraham BP. Epidemiology and risk factors of nonalcoholic fatty liver disease among patients with inflammatory bowel disease. Inflamm Bowel Dis 2017;23:998-1003.

27. Sartini A, Gitto S, Bianchini M, et al. Non-alcoholic fatty liver disease phenotypes in patients with inflammatory bowel disease. Cell Death Dis 2018;9:87.

28. Jamali R, Biglari M, Seyyed Hosseini SV, Shakouri Rad A, Kosari F. The correlation between liver fat content and ulcerative colitis disease severity. Acta Med Iran 2017;55:333-339.

29. Principi M, Iannone A, Losurdo G, et al. Nonalcoholic fatty liver disease in inflammatory bowel disease: prevalence and risk factors. Inflamm Bowel Dis 2018;24:1589-1596.

30. Tomita K, Tamiya G, Ando S, et al. Tumour necrosis factor alpha signalling through activation of Kupffer cells plays an essential role in liver fibrosis of non-alcoholic steatohepatitis in mice. Gut 2006;55:415-424.

31. Barbuio R, Milanski M, Bertolo MB, Saad MJ, Velloso LA. Infliximab reverses steatosis and improves insulin signal transduction in liver of rats fed a high-fat diet. J Endocrinol 2007;194:539-550.

32. Koca SS, Bahcecioglu IH, Poyrazoglu OK, Ozercan IH, Sahin K, Ustundag B. The treatment with antibody of TNF-alpha reduces the inflammation, necrosis and fibrosis in the non-alcoholic steatohepatitis induced by methionine- and choline-deficient diet. Inflammation 2008;31:91-98.

33. Zein CO, Yerian LM, Gogate P, et al. Pentoxifylline improves nonalcoholic steatohepatitis: a randomized placebo-controlled trial. Hepatology 2011;54:1610-1619.

34. Li W, Zheng L, Sheng C, Cheng X, Qing L, Qu S. Systematic review on the treatment of pentoxifylline in patients with non-alcoholic fatty liver disease. Lipids Health Dis 2011;10:49.

35. Lapumnuaypol K, Kanjanahattakij N, Pisarcik D, Thongprayoon C, Wijarnpreecha K, Cheungpasitporn W. Effects of inflammatory bowel disease treatment on the risk of nonalcoholic fatty liver disease: a meta-analysis. Eur J Gastroenterol Hepatol 2018;30:854-860.

36. Thin LW, Lawrance IC, Spilsbury K, Kava J, Olynyk JK. Detection of liver injury in IBD using transient elastography. J Crohns Colitis 2014;8:671-677.

37. Poynard T, Ratziu V, Naveau S, et al. The diagnostic value of biomarkers (SteatoTest) for the prediction of liver steatosis. Comp Hepatol 2005;4:10.

38. Poynard T, Ratziu V, Charlotte F, et al; CYTOL study group. Diagnostic value of biochemical markers (NashTest) for the prediction of non alcoholo steato hepatitis in patients with nonalcoholic fatty liver disease. BMC Gastroenterol 2006;6:34.

39. Ratziu V, Massard J, Charlotte F, et al; CYTOL study group. Diagnostic value of biochemical markers (FibroTest-FibroSURE) for the prediction of liver fibrosis in patients with non-alcoholic fatty liver disease. BMC Gastroenterol 2006;6:6. 


\section{Supplementary Table}

Supplementary Table 1 Study questionnaire

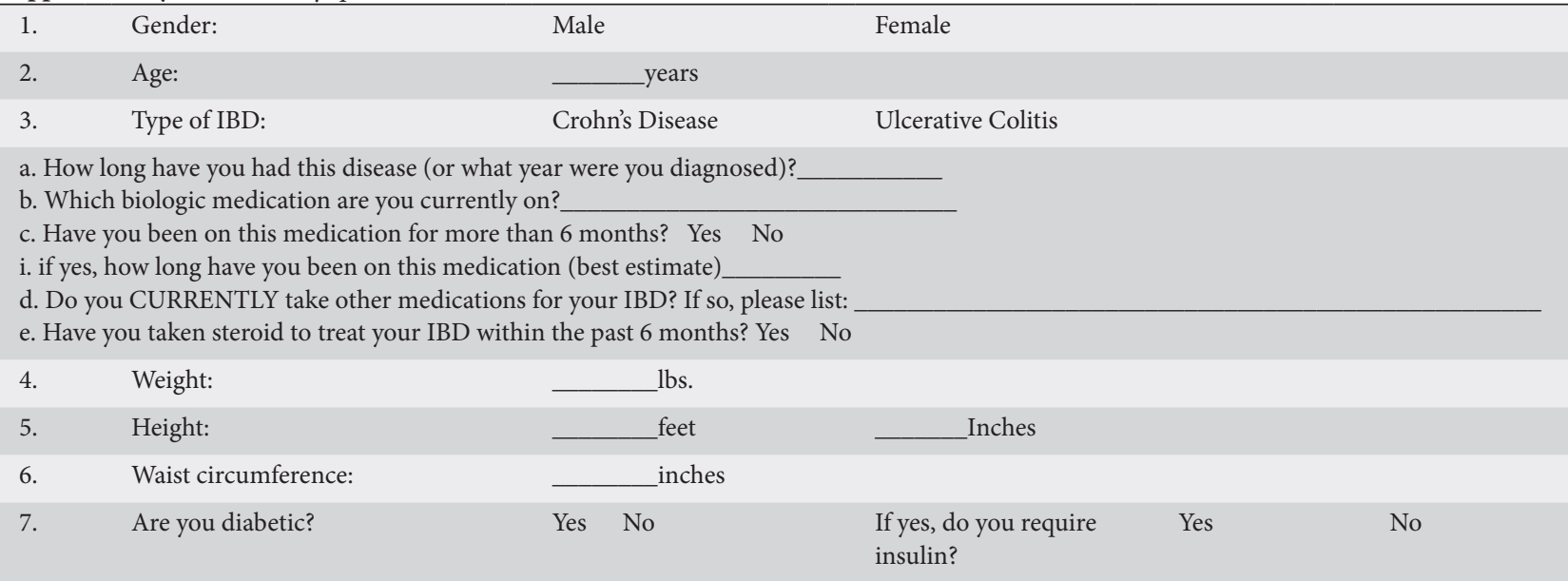

8. Do you have any personal history of liver disease?

Yes

No

a. If yes, please explain

9. Alcohol use: ON AVERAGE, how many alcoholic drinks do you consume in a week? Note: an alcoholic drink includes a $12 \mathrm{oz} \mathrm{serving}$ of beer (normal size bottle or can), a $4 \mathrm{oz}$ glass of wine, or a $1.5 \mathrm{oz}$ (or shot) of hard liquor (such as vodka, whisky, or rum):
$0-3$
$4-7$
8-14
$15-21$
$22-30$
$>30$

10. Crohn's Disease Activity Index (If you have ulcerative colitis, please skip to question 11).

a. Over the last 7 days: ON AVERAGE, how many bowel movements do you have per day?

b. On a scale of 0 to 3 , where 0 is no pain and 3 is severe pain, please rate, ON AVERAGE, how much abdominal pain you have had daily over the past week?

c. Consider your general sense of well being over the past week. On a scale of 0 to 4 , where 0 is feeling well and 4 is feeling terrible, rate your AVERAGE daily score:

d. Please check which complications of Crohn's Disease you have CURRENTLY:

i. Arthritis or joint pains. Yes No

ii. Iritis or uveitis (inflammation of your eyes). Yes No

iii. Ulcers of other lesions in your mouth or on your skin. Yes No

iv. Perirectal disease such as anal fissure, fistulas or abscesses. Yes No

v. Other fistulas. Yes No

vi. Body temperature greater than $37.8 \mathrm{C}(100.0 \mathrm{~F})$. Yes No

e. Have you used over the counter anti-diarrheal drugs in the past 7 days? Yes No

f. Have you noticed an abdominal mass recently? Yes Maybe/questionable No

11. Simple Clinical Colitis Activity Index (FOR ULCERATIVE COLITIS PATIENTS ONLY). Answer all questions based on your symptoms and how you have been feeling over the past ONE WEEK.

a. On average, how many bowel movements do you have per day (over 24 hours)?

$\begin{array}{llll}0-3 & 4-6 & 7-9 & 10+\end{array}$

b. On average, how many bowel movements do you have at night?

$0 \quad 1-3 \quad 3+$

c. When you need to have a bowel movement, how quickly do you need to go (urgency)?

$$
\text { No rush Hurry Immediately Incontinence }
$$

d. How frequently are you noticing blood in your stool, and how much?

No blood Small traces Occasionally bloody Usually bloody

e. Please rate your general well being over the past week.

$\begin{array}{llll}\text { Very well Slightly below par } & \text { Poor } & \text { Very poor } & \text { Terrible }\end{array}$

f. During the past week, have you had any of the following?

i. Pyoderma gangrenosum (oozing ulcers, usually on the legs). Yes No

ii. Erythema nodosum (red, swollen bumps, typically on the shins). Yes No

iii. Uveitis (red, painful eyes). Yes No

iv. Arthritis or Joint pains. Yes No 